\title{
ПРИМЕНЕНИЕ СИСТЕМНОГО АНАЛИЗА ДЛЯ ОБЕСПЕЧЕНИЯ ЭКСПЛУАТАЦИОННОЙ НАДЁЖНОСТИ ЭЛЕКТРИЧЕСКИХ МАШИН В АЛМАЗОДОБЫВАЮЩЕЙ ПРОМЫШЛЕННОСТИ
}

Однокопылов Георгий Иванович',

OGlz@ya.ru

\author{
Дементьев Юрий Николаевич 1 \\ Шевчук Владислав Алексеевич', \\ vashevchuk@tpu.ru
Национальный исследовательский Томский политехнический университет, Россия, 634050, г. Томск, пр. Ленина, 30.

\begin{abstract}
Актуальность. В последнее время в алмазодобывающей промышленности наметилась тенденция добычи полезных ископаемых шахтным методом. В связи с этим широкое распространение получил привод на основе электрических машин постоянного и переменного тока. Внеплановая остановка горного оборудования приводит к ухудшению экономических показателей и дополнительным расходам на ремонт. Внезапный выход из строя проходческого оборудования приводит к техногенным катастрофам и ставит под угрозу безопасность рабочего персонала. Поэтому обеспечение должного уровня эксплуатационной надёжности горного оборудования является важнейшей задачей для алмазодобывающего комплекса. Чтобы минимизировать время простоя и исключить внезапные отказы необходима своевременная диагностики, ремонт с применением современных технологий, а также постоянное совершенствование системы технического обслуживания и ремонта. На сегодняшний день применение системного анализа в области надёжности электрических машин не получило необходимого развития. Парные сравнения, применяемые в системном анализе, позволяют анализировать критерии, не поддающиеся количественной оценке.

Цель: определить наиболее эффективные пути решения проблемы обеспечения должного уровня эксплуатационной надёжности электрических машин режущего органа проходческого комбайна АМ-75 в алмазодобывающей промышленности методом анализа иерархий.
\end{abstract}

объект: электропривод режущего органа проходческого комбайна АМ-75, эксплуатируемый в условиях алмазодобывающего комплекса.

Методы: системный анализ, метод анализа иерархий, метод экспертных оценок.

Результаты. С помощью метода анализа иерархий выявлен критерий, оказывающий наиболее сильное влияние на уровень эксплуатационной надёжности электропривода режущего органа проходческого комбайна АМ-75, иерархично представлено дерево целей для решения проблемы поддержания эксплуатационной надёжности электрических машин комбайнов.

\section{Ключевые слова:}

Системный анализ, метод анализа иерархий, дерево целей, проходческий комбайн,

электрические машины, эксплуатационная надёжность, алмазодобывающая промышленность.

\section{Введение}

Технологическое оборудование алмазодобывающего комплекса г. Мирный относится к категории опасных производственных объектов с риском техногенных аварий и необходимостью снижения невосполнимых экономических потерь. Юридической основой обеспечения промышленной безопасности служит Постановление Правительства РФ от 28.03.2001 № 2410 мерах обеспечения на территории РФ промышленной безопасности опасных производственных объектов (с изменениями от 1 февраля 2005 г. и 22 апреля 2009 г.). Обеспечить промышленную безопасность опасного производственного объекта, имеющего в своем составе электропривод, возможно на основе обеспечения живучести [1-8] с применением различных видов резервирования, в том числе с повышением эксплуатационной надежности [9-20] на основе анализа работы оборудования с учетом рассмотрения нормативных и сверхнормативных режимов эксплуатации [21-24].
Обеспечение должного уровня эксплуатационной надёжности электрических машин (ЭМ) является сложной задачей, так как для разных этапов жизненного цикла оборудования характерны разные методы её решения.

В алмазодобывающей промышленности задачу обеспечения эксплуатационной надёжности предлагается рассматривать как упорядоченную иерархическую структуру критериев для достижения необходимой надёжности. В работах $[25,26]$ выявлено, что в алмазодобывающем комплексе г. Мирный наиболее подвержены отказам двигатели режущего органа проходческих комбайнов АМ-75. Комбайны эксплуатируются в условиях подземной разработки, где непрерывное воздействие оказывают такие факторы, как запылённость, влажность, сильные колебания температуры. Немаловажное значение имеет и многолетнемерзлые породы, оказывающие дополнительное влияние на бурильные части проходческого оборудования, поскольку нормативных документов по разработке подзем- 
ных месторождений в условиях многолетней мерзлоты нет и необходимые расчёты по надёжности оборудования производятся на основании производственного опыта. Задача обеспечения должного уровня эксплуатационной надёжности ЭМ режущего органа проходческого комбайна AM-75 стоит достаточно остро, поскольку его внезапный выход из строя приводит к невосполнимым экономическим убыткам и ставит безопасность рабочего персонала под угрозу. Методы системного анализа позволяют выявить такие критерии, которые наибольшим образом влияют на главную цель.

\section{Теоретическая часть}

Системный анализ - это процесс изучения поставленной задачи с целью определения действий или мероприятий (критериев системы), которые позволят решить её эффективным способом. Системный анализ нашёл своё применение в разработке искусственных систем (социальных, технических, экономических и др.), то есть в системах, где важную роль играет человек. Однако наиболее широкое распространение получил в области, связанной с принятием и обоснованием решений в проектировании [27, 28].

На сегодняшний день среди методов системного анализа большое распространение получили системы поддержки принятия решений. Количественные методы экспертных оценок, такие как метод анализа иерархий (МАИ) или метод Дельфи, дают более правдоподобные экспертные оценки при принятии решений [28].

Метод анализа иерархий является математическим аппаратом системного анализа для принятия решений в задачах, не имеющих определённо верного решения. С его помощью эксперт, основываясь на личном опыте, знаниях и понимании проблемы, смог бы решить её. Смысл метода заключается в получении парных матриц из критериев системы с их последующим сравнением. Эти критерии составляют связанные между собой уровни иерархии, которые могут группироваться в несвязные множества и таким образом структурировать сложные задачи. В МАИ входят следующие этапы указанного процесса [27-32]:

- формирование структуры проблемы со связями ее критериев;

- моделирование критериальных оценок с экспертными предпочтениями;

- синтез правила решения с установленными предпочтениями на альтернативных множествах.

Преимуществами МАИ являются [33-36]:

- при сравнении экспертом факторов по парам позволяет концентрироваться на конкретной проблеме и исключает необходимость в постоянной проверке групп однородных факторов;

- в случаях, когда необходимо скорректировать факторы, пересчитываются только новые значения или же удаляются строки и столбцы, соответствующие изъятым факторам;
- использует вербально-числовую шкалу Саати, которая позволяет оценивать факторы, выражаемые в разных размерностях;

- позволяет проверять результаты экспертов на согласованность, что является удобством при автоматизации процесса.

К недостаткам МАИ следует отнести [28, 34]:

- необходимость обработки большого количества информации от экспертов;

- не позволяет проверить оценку эксперта на согласованность, если критериев больше 15 . В таких случаях необходимо дальнейшее разделение на подгруппы;

- оценки являются субъективными, поскольку эксперт опирается исключительно на собственные знания и опыт.

Применение МАИ в сложных задачах выбора является лучшим решением, поскольку имеет ряд преимуществ перед методом Дельфи, таких как $[28,29]:$

- наличие конкретного математического аппарата; - поддержка взаимодействий и дискуссий, что способствует появлению новых решений. При этом все мнения учитываются при расчётах;

- разделение поставленной задачи на более простые.

Иерархия - это тип системы, при которой его элементы могут группироваться в множества, несвязанные между собой. Однако элементы одних групп находятся под влиянием других и так далее [35].

Задача обеспечения должного уровня эксплуатационной надёжности ЭМ режущего органа комбайна в алмазодобывающей промышленности является достаточно сложной и обширной. Для её успешного выполнения необходимо не только учитывать ряд мероприятий, которые оказывают как прямое, так и косвенное влияние, но и подобрать экспертов, имеющих достаточный уровень знаний, для выставления адекватных оценок при парных сравнениях. В практике не редки случаи, когда количество элементов и их связей настолько велико, что затрудняет анализ. В таких случаях необходимо разделение системы на подсистемы.

\section{Практическая часть}

В работе [37] представлено дерево целей для задачи повышения әффективности эксплуатации и обслуживания ЭМ в горнодобывающем комплексе. Однако слишком большое количество критериев не позволяет дать точную оценку, так как это усложняет задачу и приводит к рассогласованности матриц [27, 28, 30,38]. На рис. 1 в виде дерева целей представлены критерии, наиболее влияющие на обеспечение заданного уровня эксплуатационной надёжности ЭМ режущего органа проходческого комбайна AM-75. Первый уровень является уровнем общей цели и представляет собой основную задачу. В нашем случае это обеспечение эксплуатационной надёжности ЭМ в алмазодобывающей промышленности. На втором уровне были 
расположены критерии, влияющие на прирост уровня эксплуатационной надёжности. На третьем и четвёртом уровне были классифицированы мероприятия по повышению эксплуатационной надёжности, где 1.1.1.1. - Ремонт по техническому состоянию; 1.1.1.2. - Фирменный метод ТО и ремонта; 1.1.2.1. - ТО по событию; 1.1.2.2. - ТО по регламенту; 1.1.2.3. - Т0 по состоянию; 1.1.3.1. Соблюдение эксплуатационных режимов; 1.1.3.2. - Энергосберегающие мероприятия; 1.1.3.3. - Модернизация оборудования; 1.1.4.1. Средства и сооружения для выполнения ТО и ремонта; 1.2.1.1. - Тестовое диагностирование; 1.2.1.2. - Рабочее диагностирование; 1.2.1.3. - Экспресс-диагностирование; 1.3.1.1. - Параметрическое оценивание надёжности; 1.3.1.2. - Непараметрическое оценивание надёжности; 1.3.1.3. Определение работоспособного интервала времени с заданной вероятностью; 1.3.1.4. - Определение вероятности работоспособности в определенный интервал времени.

На примере расчёта критериев второго уровня относительно главной цели рассмотрим работу механизма МАИ [27, 28]. После построения дерева целей экспертами заполняется матрица парных сравнений порядка $n$ ( $n$ - количество критериев). Для данной работы экспертные оценки были получены от инженеров алмазодобывающего комплекса в г. Мирный (Якутия). Такая матрица является квадратной, обратно симметричной с единицами на главной диагонали. Сравнение происходит между критериями в строчках и критериями в столбцах по правилам [39-41]:

$$
a_{i j}=\alpha ; a_{j i}=1 / \alpha ; a_{i j}=a_{j i}=1,
$$

где $\alpha$ - оценка эксперта по 9-балльной шкале, где 1 означает равную важность, а 9 - полное превосходство одного критерия над другим.

Если рассматривается $n$ критериев, то всего возможно наличие $\left(n^{2}-n\right) / 2$ значащих сочетаний [35, 42-44]. Например, при сравнении пары критериев 1.1. и 1.3. (табл. 1) эксперт посчитал, что первый умеренно превосходит второй, что соответствует цифре 3 в табл. 1. Это значит, что паре критериев 1.3. и 1.1. автоматически соответствует значение $1 / 3$. Далее по формуле (1) находим геометрическую сумму каждой строки и по (2) их сумму:

$$
\begin{gathered}
a_{i}=\sqrt[n]{\prod_{j=1}^{n} x_{i j}}, \\
\sum a_{i}=a_{1}+a_{2}+\ldots+a_{n} .
\end{gathered}
$$

Компонентами нормализованного вектора приоритетов являются отношения геометрической суммы строк на общую сумму (3):

$$
\begin{gathered}
V_{i}=a_{i} / \sum a_{i}, \\
V_{1}=0,25 ; \quad V_{2}=0,65 ; V_{3}=0,1 .
\end{gathered}
$$

В алгоритм МАИ заложен расчёт индекса согласованности (ИС), который необходим для проверки оценок эксперта на согласованность [28, 40]. Для

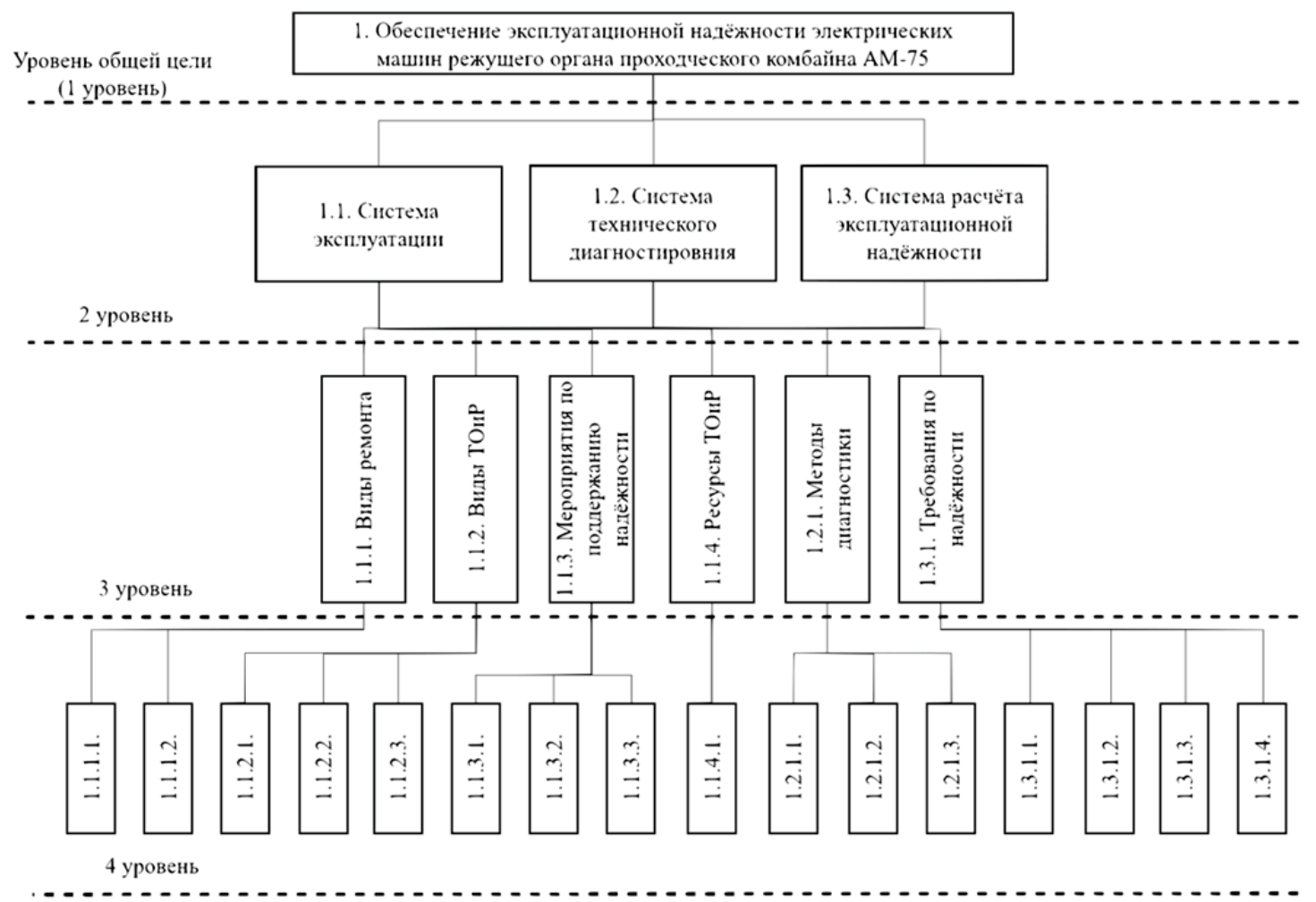

Pис. 1. Дерево целей для задачи по обеспечению эксплуатащионной надёжности ЭМ режущего органа проходческого комбайна АМ-75

Fig. 1. Tree of goals for the task of ensuring the operational reliability of the AM-75 roadheader cutting body electrical machine 
таблица 1. Величины случайной согласованности

Table $1 . \quad$ Values of random consistency

\begin{tabular}{|c|c|c|c|c|c|c|c|c|c|c|c|c|c|c|c|}
\hline$n$ & 1 & 2 & 3 & 4 & 5 & 6 & 7 & 8 & 9 & 10 & 11 & 12 & 13 & 14 & 15 \\
\hline $\mathrm{CC}$ & 0 & 0 & 0,58 & 0,9 & 1,12 & 1,24 & 1,32 & 1,41 & 1,45 & 1,49 & 1,51 & 1,48 & 1,56 & 1,57 & 1,59 \\
\hline
\end{tabular}

начала нужно произвести расчёт максимального собственного значения матрицы $\lambda_{\max }$ по (4). Чем ближе значение $\lambda_{\max } \kappa$ порядку матрицы $n$, тем выше будет её значение согласованности $\left(\lambda_{\max } \geq n\right)$ [45].

$$
\lambda_{\text {max }}=V_{1} R_{1}+V_{2} R_{2}+\ldots+V_{n} R_{n},
$$

где $R_{n}$ - сумма элементов $n$-столбца.

Расчёт ИС производится по следующей формуле (5):

$$
\text { ИС }=\frac{\lambda_{\max }-n}{n-1} .
$$

Значение ИС необходимо сравнить с величинами средней согласованности (СC), которые были получены в работе [28] для матриц порядка от 1 до 15 (табл. 1). Отношение ИС к СС называется отношением согласованности (ОС) (6).

$$
\mathrm{OC}=\frac{\mathrm{UC}}{\mathrm{CC}} .
$$

Если значения величин ОС и ИС меньше или равны 0,1 , то матрица является согласованной. Однако в системах с большим количеством критериев допускается величина $\mathrm{OC} \leq 0,2[27,28,35,46,47]$.

Таблица 2. Матрица парных сравнений критериев второго уровня по отношению к главной цели

\begin{tabular}{|c|c|c|c|c|c|c|c|c|}
\hline & 1.1. & 1.2. & 1.3. & $a_{i}$ & $\Sigma a_{i}$ & $\lambda_{\text {max }}$ & $\begin{array}{c}\text { ИС } \\
\text { Consistency } \\
\text { index }\end{array}$ & $\begin{array}{c}\mathrm{OC} \\
\text { Consistency } \\
\text { relation }\end{array}$ \\
\hline 1.1. & 1 & $1 / 3$ & 3 & 1 & \multirow{3}{*}{4} & \multirow{3}{*}{3,02} & \multirow{3}{*}{0,01} & \multirow{3}{*}{0,02} \\
\hline 1.2 . & 3 & 1 & 6 & 2,62 & & & & \\
\hline 1.3 . & $1 / 3$ & $1 / 6$ & 1 & 0,38 & & & & \\
\hline
\end{tabular}

Table 2. Array of pairwise comparisons of the $2^{\text {nd }}$ level criteria in relation to the main goal

Для определения наиболее важного критерия в достижении главной цели воспользуемся расчётом коэффициента относительной важности (КОВ). Для его расчёта используют методы нормирования, ранжирования, групповой экспертизы и другие [28]. В нашем случае воспользуемся методом ранжирования. Для этого необходимо в каждой строке матрицы (табл. 1) найти максимальное значение и вычесть его из каждого элемента строки. Итогом станет ранжированная матрица (табл. 3).

\begin{tabular}{|c|c|c|c|c|c|c|}
\hline & 1.1. & 1.2. & 1.3. & $a_{i}$ & $\sum a_{i}$ & KOB/Relative importance factor $\left(W_{j}\right)$ \\
\hline 1.1. & 1 & $1 / 3$ & 3 & 4,67 & \multirow{3}{*}{14,17} & 0,329 \\
\hline 1.2 . & 3 & 1 & 6 & 8 & & 0,565 \\
\hline 1.3. & $1 / 3$ & $1 / 6$ & 1 & 1,5 & & 0,106 \\
\hline
\end{tabular}

Таблица 3. Ранжированная матрица критериев второго уровня Table 3. Ranked array of the $2^{\text {nd }}$ level elements
КОВ критериев (8) есть отношение суммы элементов строки ранжированной матрицы (7) и общей суммы (2).

$$
\begin{aligned}
a_{i} & =\sum_{j=1}^{n} X_{i j} ; \\
W_{j} & =\frac{a_{i}}{\sum a_{i}} .
\end{aligned}
$$

Согласно табл. 3 , наиболее сильно влияющим критерием второго уровня является «Система технического диагностирования» с КОВ, равным 0,565. Меньшее влияние оказывают «Система эксплуатации» (КОВ равен 0,329) и «Система расчёта эксплуатационной надёжности» (КОВ равен $0,106)$. ИС и ОС меньше 0,1 , что выполняет условие согласованности. Второй уровень представляет собой разбиение главной цели на критерии, которые отображают эффективность обслуживания и эксплуатации электрических машин режущего органа комбайна. Наибольшее влияния критерия диагностики в данном случае очевидно, поскольку своевременная диагностика и ремонт ЭМ позволяют избежать внезапного выхода из строя.

По такому же алгоритму необходимо провести расчёты для третьего уровня. Однако стоит заметить, что так как вышестоящих критериев три, парное сравнение необходимо провести относительно каждого из трёх критериев.

В табл. 4-6 из критериев третьего уровня рассчитаны векторы КОВ. Для получения общего вектора КОВ необходимо матрицу из трёх векторов умножить на вектор КОВ второго уровня.
$0,22 \quad 0,172 \quad 0,187$
0,189 1.1.1.
$0,206 \quad 0,277 \quad 0,197$
$0,27 \quad 0,268 \quad 0,192$
$0,245 \quad 1.1 .2$
0,052
0,037
0,329
$0,261.1 .3$.
0,152
0,123
0,195
$\times 0,565=$
0,058
1.1.4.
0,1
$0,124 \quad 0,031$
0,14
1.2.1.
0,106 1.3.1.

Согласно общему вектору, из критериев третьего уровня наиболее сильное влияние на главную цель имеют мероприятия по поддержанию надёжности (критерий 1.1.3.) с КОВ, равным 0,26 (рис. 2). Стоит отметить, что критерий 1.1.2. «Виды ТОиР» также имеет достаточно высокий показатель КОВ $\left(W_{j}=0,245\right)$, что подтверждает сказанное выше о своевременном обслуживании. Третий уровень дерева целей, в котором были структурированы критерии четвертого уровня, является промежуточным, что в дальнейшем позволит произвести оценку только тех критериев, которые 
Таблица 4. Матрица парных сравнений критериев третьего уровня по отношению к критерию 1.1.

Table 4. $\quad$ Array of the pairwise comparisons of the $3^{\text {rd }}$ level criteria in relation to criterion 1.1.

\begin{tabular}{|c|c|c|c|c|c|c|c|c|c|c|}
\hline & 1.1.1. & 1.1.2. & 1.1.3. & 1.1.4. & 1.2.1. & 1.3 .1 & $\begin{array}{c}\text { KOB } \\
\text { Relative importance factor }\left(W_{j}\right)\end{array}$ & $\lambda_{\max }$ & $\begin{array}{c}\text { ИС } \\
\text { Consistency index }\end{array}$ & $\begin{array}{c}\text { OC } \\
\text { Consistency relation }\end{array}$ \\
\hline 1.1.1. & 1 & 1 & 1 & 4 & 1 & $1 / 2$ & 0,22 & \multirow{6}{*}{6,4} & \multirow{6}{*}{0,09} & \multirow{6}{*}{0,07} \\
\hline 1.1 .2$. & 1 & 1 & 2 & 4 & 1 & $1 / 2$ & 0,206 & & & \\
\hline 1.1.3. & 1 & $1 / 2$ & 1 & 5 & 3 & $1 / 2$ & 0,27 & & & \\
\hline 1.1.4. & $1 / 4$ & $1 / 4$ & $1 / 5$ & 1 & $1 / 3$ & $1 / 3$ & 0,052 & & & \\
\hline 1.2 .1$. & 1 & 1 & $1 / 3$ & 3 & 1 & 1 & 0,152 & & & \\
\hline 1.3 .1$. & 2 & 2 & 2 & 3 & 1 & 1 & 0,1 & & & \\
\hline
\end{tabular}

Таблица 5. Матрица парных сравнений критериев третьего уровня по отношению к критерию 1.2.

Table 5. $\quad$ Array of pairwise comparisons of the $3^{\text {rd }}$ level criteria in relation to criterion 1.2.

\begin{tabular}{|c|c|c|c|c|c|c|c|c|c|c|}
\hline & 1.1.1. & 1.1.2. & 1.1.3. & 1.1.4. & 1.2.1. & 1.3 .1 & $\begin{array}{c}\text { KOB } \\
\text { Relative importance factor }\left(W_{j}\right)\end{array}$ & $\lambda_{\text {max }}$ & $\begin{array}{c}\text { ИС } \\
\text { Consistency index }\end{array}$ & $\begin{array}{c}\text { OC } \\
\text { Consistency relation }\end{array}$ \\
\hline 1.1.1. & 1 & 3 & 4 & 5 & 7 & 9 & 0,268 & \multirow{6}{*}{6,4} & \multirow{6}{*}{0,08} & \multirow{6}{*}{0,07} \\
\hline 1.1.2. & $1 / 3$ & 1 & 3 & 4 & 7 & 7 & 0,211 & & & \\
\hline 1.1 .3$. & $1 / 4$ & $1 / 3$ & 1 & 3 & 4 & 5 & 0,176 & & & \\
\hline 1.1.4. & $1 / 5$ & $1 / 4$ & $1 / 3$ & 1 & 3 & 4 & 0,163 & & & \\
\hline 1.2 .1$. & $1 / 7$ & $1 / 7$ & $1 / 4$ & $1 / 3$ & 1 & 3 & 0,141 & & & \\
\hline 1.3 .1$. & $1 / 9$ & $1 / 7$ & $1 / 5$ & $1 / 4$ & $1 / 3$ & 1 & 0,042 & & & \\
\hline
\end{tabular}

Таблица 6. Матрица парных сравнений критериев третьего уровня по отношению к критерию 1.3.

Table 6. $\quad$ Array of the pairwise comparisons of the $3^{\text {rd }}$ level criteria in relation to criterion 1.3.

\begin{tabular}{|c|c|c|c|c|c|c|c|c|c|c|}
\hline & 1.1.1. & 1.1.2. & 1.1.3. & 1.1.4. & 1.2.1. & 1.3.1. & $\begin{array}{c}\text { KOB } \\
\text { Relative importance factor }\left(W_{j}\right)\end{array}$ & $\lambda_{\max }$ & $\begin{array}{c}\text { ИС } \\
\text { Consistency index }\end{array}$ & $\begin{array}{l}\text { OC } \\
\text { Consistency relation }\end{array}$ \\
\hline 1.1.1. & 1 & $1 / 4$ & $1 / 5$ & $1 / 3$ & 2 & 6 & 0,187 & \multirow{6}{*}{6,5} & \multirow{6}{*}{0,1} & \multirow{6}{*}{0,08} \\
\hline 1.1 .2 . & 4 & 1 & $1 / 3$ & 3 & 4 & 8 & 0,197 & & & \\
\hline 1.1 .3$. & 5 & 3 & 1 & 4 & 5 & 9 & 0,192 & & & \\
\hline 1.1.4. & 3 & $1 / 3$ & $1 / 4$ & 1 & 3 & 7 & 0,195 & & & \\
\hline 1.2.1. & $1 / 2$ & $1 / 4$ & $1 / 5$ & $1 / 3$ & 1 & 6 & 0,198 & & & \\
\hline 1.3 .1$. & $1 / 6$ & $1 / 8$ & $1 / 9$ & $1 / 7$ & $1 / 6$ & 1 & 0,031 & & & \\
\hline
\end{tabular}

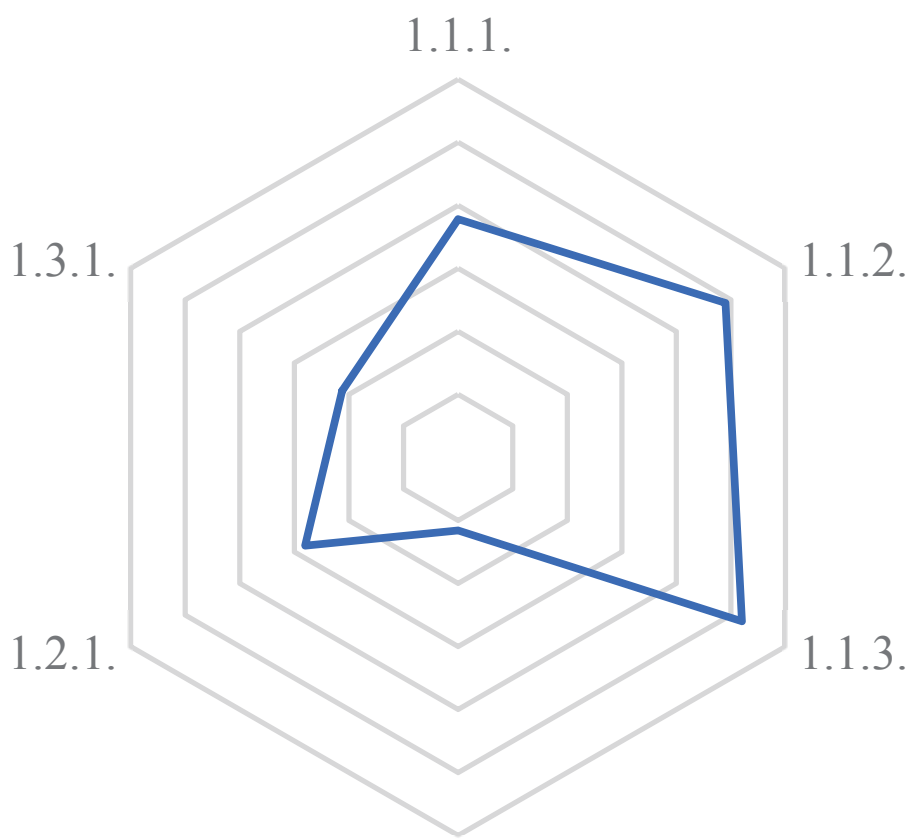

1.1.4.

1.1.1. - Виды ремонта;

1.1.2. - Виды ТОиР;

1.1.3. - Мероприятия по

поддержанию надёжности;

1.1.4. - Ресурсы ТОиР;

1.2.1. - Методы диагностикг

1.3.1. - Требования по

надёжности.

Рис. 2. Диаграмма значимости критериев третьего уровня

Fig. 2. Chart of the $3^{\text {rd }}$ level criteria 
имеют непосредственное отношение к весомому критерию третьего уровня. Согласно дереву целей, пункт 1.1.3. разделяется на критерии: «Соблюдение эксплуатационных режимов»; «Энергосберегающие мероприятия» и «Модернизация оборудования».

Таблица 7. Матрица парных сравнений критериев четвертого уровня по отношению к критерию 1.1.3.

Table 7. Array of pairwise comparisons of the $4^{\text {th }}$ level criteria in relation to criterion 1.1.3.

\begin{tabular}{|c|c|c|c|c|c|c|c|}
\hline & 1.1.3.1. & 1.1.3.2. & 1.1.3.3. & 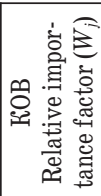 & $\lambda_{\max }$ & 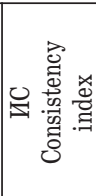 & 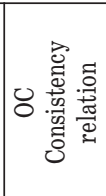 \\
\hline 1.1.3.1. & 1 & 7 & 2 & 0,336 & \multirow{3}{*}{3,05} & \multirow{3}{*}{0,03} & \multirow{3}{*}{0,05} \\
\hline 1.1.3.2. & $1 / 7$ & 1 & $1 / 7$ & 0,068 & & & \\
\hline 1.1.3.3. & $1 / 2$ & 7 & 1 & 0,596 & & & \\
\hline
\end{tabular}

Согласно полученному вектору (табл. 7), критерий «Модернизация оборудования» (с КОВ равным $0,596)$ наиболее сильно влияет на главную цель.

\section{Заключение}

Произведён подробный анализ иерархий задачи обеспечения эксплуатационной надёжности ЭМ режущего органа проходческого комбайна AM-75 в алмазодобывающей промышленности. Благодаря МАИ получена возможность систематизировать задачу, а на основе данных, полученных от экспертов, выявить наиболее эффективное решение при наличии нескольких альтернатив. В работе получены следующие результаты:

1. Согласно вектору критериев относительной важности второго уровня наиболее весомым критерием является «Система технического диагностирования» с КОВ, равным 0,565 , далее идёт «Система эксплуатации» (КОВ равен $0,329)$ и «Система расчёта эксплуатационной надёжности» (КОВ равен 0,106$)$.

\section{СПИСОК ЛИТЕРАТУРЫ}

1. Стекольников Ю.И. Живучесть систем. - СПб.: Политехника, 2002. -155 c.

2. Odnokopylov G.I., Bragin A.D. Fault tolerant vector control of induction motor drive (Article number 012015) // IOP Conference Series: Materials Science and Engineering. - 2014. - V. 66. №. . 1. - P. 1-6.

3. Odnokopylov G.I., Rozaev I.A. Formation of failure matrix and failure-free control algorithm for multi-sectioned Switched-reluctance drive (Article number 012035) / IOP Conference Series: Materials Science and Engineering. - 2014. - V. 66. - № 1. P. 1-7.

4. Odnokopylov G.I., Bragin A.D. Mathematical model of brushless DC motor in phase loss operation mode // Applied Mechanics and Materials. - 2015. - V. 698. - P. 24-29.

5. Odnokopylov G.I., Bragin A.D. Algorithms of fault tolerant control of induction motor electric drive in phase loss operate mode // 2015 International Siberian Conference on Control and Commu-
2. Для определения наиболее весомого критерия третьего уровня из трёх векторов каждого критерия второго уровня был получен общий вектор КОВ, согласно которому наиболее весомым критерием является «Мероприятия по поддержанию надёжности» с КОВ, равным 0,26.

3. Критерий «Модернизация оборудования» (c КОВ, равным 0,596) наиболее сильно влияет на главную цель. В связи с агрессивной средой эксплуатации элементы ЭМ комбайна подвергаются постоянному износу даже во время простоя. От повышенной влажности $(80-98 \%)$ происходит коррозия металлических деталей, которая ухудшает электрические свойства. Изза повышенной запылённости (70-695 мг/м $\left.{ }^{3}\right)$ происходит загрязнение изоляции, что приводит к дальнейшему её пробою. Пыль забивается в подшипниковый узел и, смешиваясь со смазкой, вызывает быстрый износ электрической машины. Длительная работа в условиях высокой запылённости приводит к образованию слоя пыли на обмотках, что приводит к перегреву.

4. При расчёте все оценки экспертов были подвергнуты проверке суждений на согласованность, результатом которой во всех случаях стали низкие показатели значений индекса согласованности и отношения согласованности (ниже 0,1). Это говорит о компетентности экспертов и позволяет сделать выводы на основе их оценок.

Результаты, полученные в работе, показывают, что в случае с проходческим комбайном АM-75 необходимо не только усовершенствование системы технического облуживания и ремонта, но и модернизация самих узлов, наиболее подверженных факторам внешней среды. Поскольку метод анализа иерархий является универсальным, его целесообразно применить и к другим типам производства. Однако в этом случае необходимо пересмотреть дерево целей, так как для разного типа оборудования характерны разные критерии.

nications (SIBCON): Proc. - Omsk, May 21-23, 2015. - Novosibirsk: IEEE Russia Siberia Section, 2015. - P. 1-5.

6. Odnokopylov G.I., Rozaev I.A. Fault-tolerant control of switched-reluctance drive in emergency modes // 2015 International Siberian Conference on Control and Communications (SIBCON): proceedings, Omsk, May 21-23, 2015. - Novosibirsk: IEEE Russia Siberia Section, 2015. - P. 1-6.

7. Odnokopylov G.I., Rozaev I.A. Fault-tolerant control algorithms of switched-reluctance motor drive in open-phase modes // 2016 The $11^{\text {th }}$ International Forum on Strategic Technology (IFOST): Proc. - Novosibirsk, June 1-3, 2016. - Novosibirsk: IEEE Russia Siberia Section, 2016. - P. 140-144.

8. Ткачук Р.Ю., Глазырин А.С. Принцип построения отказоустойчивой системы управления асинхронным электроприводом // Известия Томского политехнического университета. 2012. - T. 321. - № 5. - C. 105-109.

9. Almashakbeh A.S., Prus V., Zagirnyak M. Models for electric machine reliability prediction at variation of the condition of ba- 
sic structural units // Przeglad elektrotechniczny. - 2017. V. 1 (93). - P. 117-120.

10. Muravleva 0.0., Tyuteva P.V. Operation features of the improved induction motors in the variable speed drive of pump units // EUROCON 2009. - St. Petersburg, May 18-23 2009. P. 703-708.

11. Muravleva 0.0. Resource saving prospects based on accuracy theory // EUROCON 2007 - The International Conference on Computer as a Tool. - Warsaw, Poland, September 9-12, 2007. P. $1845-1850$.

12. Glowacz A., Glowacz Z. Diagnostics of stator faults of the singlephase induction motor using thermal images, MoASoS and selected classifiers // Measurement. - 2016. - V. 93. - P. 86-93.

13. Electrical \& mechanical diagnostic indicators of wind turbine induction generator rotor faults / D. Zappalá, N. Sarma, S. Djurović, C.J. Crabtree, A. Mohammad, P.J. Tavner // Renewable energy. - 2019. - V. 131. - P. 14-24.

14. Sakhara S., Saad S. Diagnosis and detection of short circuit in asynchronous motor using three-phase model // International journal of system assurance engineering and management. 2017. - V. 8. - P. 308-317.

15. A fault-tolerant control strategy for switched reluctance motor drive for electric vehicles under short-fault condition / M. Ma, R. Wang, F. Li, J. Wang, S. Yang // Microelectronics reliability. 2018. - V. 88-90. - P. 1221-1225.

16. Performance comparison of fault-tolerant three-phase induction motor drives considering current and voltage limits / M. Tousizadeh, H.S. Che, J. Selvaraj, N.A. Rahim, B.-T. Ooi // IEEE Transactions on Industrial Electronics. - 2019. - V. 66 (4). P. 2639-2648

17. Шевчук В.А., Муравлев 0.П. Анализ вероятности безотказной работы электрических машин в алмазодобывающей промышленности // Горное оборудование и электромеханика. 2018. - № 4 (138). - C. 39-46.

18. Brushless DC motor torque estimation based on nonlinear partial least square method / Z. Zhiyong, Z. Fei, L. Zhiqiang, Z. Xin // Transactions of China electrotechnical society. -2017 . - V. 32. - P. 62-69.

19. Analysis, design and dynamic simulation of novel limited swing angle torque permanent magnet motor for high voltage circuit breaker application / G. Zeng, X. Yang, S. Zhao, H. Yin, Y. Pei, J. Cao // Energies. - 2018. - V. 11 (10). - P. 1-18.

20. Meng L., Liu Z. Fault simulation and diagnosis for vector control of AC motor drive // IEEJ Transactions on Electrical and Electronic Engineering. - 2016. - V. 11 (5). - P. 618-623.

21. Zyuzev A.M., Bubnov M.V. Model for Sucker-Rod Pumping Unit Operating Modes Analysis Based on SimMechanics Library // Journal of Physics: Conference Series. - 2018. - V. 944 . - P. 1-7.

22. Зюзев А.М., Метельков В.П. 0 проблеме перегрева обмотки ротора асинхронных двигателей в пусковых режимах высокоинерционных электроприводов нефтегазовой и горной промышленности // Известия Томского политехнического университета. Инжиниринг георесурсов. - 2018. - Т. 329. - № 7. C. $96-103$.

23. Зюзев А.М., Метельков В.П. Учет колебаний температуры обмотки статора асинхронного двигателя при проверке по нагреванию в повторно-кратковременном режиме // Известия Томского политехнического университета. Инжиниринг георесурсов. -2015 . - Т. 326. - № 4. - С. 146-153.

24. Zyuzev A.M., Kostylev A.V., Stepanyuk D.P. A soft-startup device for an induction motor with supply-network voltage control // Russian Electrical Engineering. - 2014. - V. 85 (9). - P. 586-590.

25. Ruan L., Chen J., Gu G. Different Influence of Cooling Method to Stator Bar Insulation Characteristics in Pumped Storage Units // Diangong Jishu Xuebao/Transactions of China Electrotechnical Society. - 2017. - V. 32 (14). - P. 246-251.

26. Shevchuk V.A., Muravlev 0.P., Stolyarova 0.0. Survival analysis of a.c. machines in the diamond industry using the Kaplan-
Meier estimator // MATEC Web of Conferences. - 2017. V. 141. - P. 1-4.

27. The use of a special software for induction motor diagnostics in the diamond industry / V.A. Shevchuk, O.P. Muravlev, 0.0. Stolyarova, V.P. Shevchuk // MATEC Web of Conferences. - 2017. V. 91. - P. 1-4.

28. Саати Т. Принятие решений. Метод анализа иерархии. - М.: Радио и связь, 1993. - 320 с.

29. Saaty T.L., Vargas L.G. Models, Methods, Concepts \& Applications of the Analytic Hierarchy Process. - Boston: Springer US, 2001. $-333 \mathrm{p}$.

30. Андрейчиков А.В., Андрейчикова 0.Н. Анализ, синтез, планирование решений в экономике. - М.: Финансы и статистика, 2000. - 368 c.

31. Перегудов Ф.И., Тарасенко Ф.П. Основы системного анализа. Томск: Изд-во НТЛ, 2001. - 396 с.

32. Brunelli M. Introduction to the Analytic Hierarchy Process. New York: Springer, 2015. - $83 \mathrm{p}$.

33. Антонов А.В. Системный анализ. - М.: Высшая школа, 2006. $454 \mathrm{c.}$

34. Тутыгин А.Г., Коробов В.Б. Преимущества и недостатки метода анализа иерархий // Известия Российского государственного педагогического университета им. А.И. Герцена. - 2010. № 122. - С. 108-115.

35. Деордица Ю.С. Интеллектуальные системы поддержки принятия решений. - Луганск: ВНУ, 2005. - 64 c.

36. Hierarchical model of assessing and selecting experts / T.Y. Chernysheva, M.A. Korchuganova, V.V. Borisov, S.L. Minkov // IOP Conference Series: Materials Science and Engineering. - 2016. - V. 127. - P. 1-6.

37. He M.X., An X. Information security risk assessment based on analytic hierarchy process // Indonesian Journal of Electrical Engineering and Computer Science. - 2016. - V. 1(3). - P. 656-664.

38. Гусев В.В., Муравлев 0.П., Шевчук В.П. Системный анализ эффективности функционирования электрических машин в горнодобывающем комплексе // Известия Томского политехнического университета. - 2009. - Т. 314. - № 4. - С. 74-78.

39. Fei S., Xiangdong L., Yali F. Safety evaluation for bridge crane based on FTA and AHP // MATEC Web of Conferences. - 2018. V. 207. - P. 1-4.

40. Макарова И.Л. Анализ методов определения весовых коэффициентов в интегральном показателе общественного здоровья // Символ науки. - 2015. - № 7. - С. 87-95.

41. Emrouznejad A., Ho W. Fuzzy Analytic Hierarchy Process. - Boca Raton: CRC Press, 2018. $-407 \mathrm{p}$.

42. Greco S., Ehrgott M., Figueira J.R. Multiple Criteria Decision Analysis: State of the Art Surveys. - New York: Springer, 2016. - $1346 \mathrm{p}$.

43. Intelligent collaborative support system for AHP-group decision making / G. Kou, X. Chao, Y. Peng, L. Xu, Y. Chen // Studies in Informatics and Control. - 2017. - V. 26 (2). - P. 131-142.

44. Research on comprehensive evaluation system of urban energy data based on AHP method / X.D. Ma, Q. Sun, J.M. Zhang, P. He // IOP Conference Series: Materials Science and Engineering. 2018. - V. 382 (5). - P. 1-7.

45. Choosing the Optimal Technology to Rehabilitate the Pipes in Water Distribution Systems Using the AHP Method / I. Așchilean, G. Badea, I. Giurca, G.S. Naghiu, F.G. Iloaie // Energy Procedia. - 2017. - V. 112. - P. 19-26.

46. Evans G.W. Multiple Criteria Decision Analysis for Industrial Engineering: Methodology and Applications. - Boca Raton: CRC Press, 2018. $-467 \mathrm{p}$.

47. Khazaii J. Advanced Decision Making for HVAC Engineers: Creating Energy Efficient Smart Buildings. - Switzerland: Springer International Publishing, 2016. - 191 p.

Поступила: 30.01.2019 2. 


\section{Информация об авторах}

Однокопылов Г.И., доктор технических наук, доцент отделения электроэнергетики и электротехники Национального исследовательского Томского политехнического университета.

Дементьев Ю.Н., кандидат технических наук, профессор отделения электроэнергетики и электротехники Национального исследовательского Томского политехнического университета.

Шевчук B.A., аспирант отделения электроэнергетики и электротехники Инженерной школы энергетики Национального исследовательского Томского политехнического университета. 


\title{
APPLICATION OF SYSTEM ANALYSIS FOR PROVIDING RELIABILITY OF ELECTRICAL MACHINES IN DIAMOND INDUSTRY
}

\author{
Georgy I. Odnokopylov', \\ OGIz@yandex.ru \\ Yury N. Dementyev \\ Vladislav A. Shevchuk', \\ vashevchuk@tpu.ru \\ 'National Research Tomsk Polytechnic University,
30, Lenin Avenue, Tomsk, 634050, Russia.
}

The relevance. Recently, the diamond mining industry has included a trend of mining using the mining method. In this regard, the drive based on DC motor and AC motor has become widespread. Unplanned shutdowns of mining machinery lead to economic recession and additional costs for repairs. Unplanned break of tunneling equipment leads to industrial disasters and jeopardize the safety of personnel. Therefore there is an urgent problem of providing adequate level reliability of mining machinery amongst other problems of diamond industry. Early diagnostics, repair using modern technologies and constant improvement of maintenance minimize downtime and eliminate sudden failure. Application of system analysis in reliability of electrical machines does not get necessary development. Paired comparisons applied in the system analysis allow analyzing the criteria reluctant to quantitative evaluation.

The main aim of the research is to determine the most effective ways to solve the problem of ensuring the proper level of operational reliability of electric machines of the cutting body of the AM-75 roadheader in the diamond industry by analyzing hierarchies.

object of research is the electric drive of the cutting body of the AM-75 tunnel miner, operated under conditions of the diamond-mining complex.

Methods: system analysis, hierarchy method, expert evaluation method.

Results. Using the hierarchy analysis method the authors have determined the criterion which affects most of all the reliability of electrical machines of cutting unit of road header AM-75. The paper introduces in hierarchy the objective tree for the problem of providing reliability for electrical machines of road header.

Key words:

System analysis, hierarchy analysis method, goal tree, roadheader, electrical machines, operational reliability, diamond industry.

\section{REFERENCES}

1. Stekolnikov Yu.I. Zhivuchest sistem [System survivability]. StPetersburg, Politekhnika Publ., 2002. 155 p.

2. Odnokopylov G.I., Bragin A.D. Fault tolerant vector control of induction. IOP Conference Series: Materials Science and Engineering, 2014, vol. 66, no. 1, pp. 1-6.

3. Odnokopylov G.I., Rozaev I.A. Formation of failure matrix and failure-free control algorithm for multi-sectioned Switched-reluctance drive. IOP Conference Series: Materials Science and Engineering, 2014, vol. 66, no. 1, pp. 1-7.

4. Odnokopylov G.I., Bragin A.D. Mathematical model of brushless DC motor in phase loss operation mode. Applied Mechanics and Materials, 2015, vol. 698, pp. 24-29.

5. Odnokopylov G.I., Bragin A.D. Algorithms of fault tolerant control of induction motor electric drive in phase loss operate mode. 2015 International Siberian Conference on Control and Commu nications (SIBCON): Proc. Omsk, May 21-23, 2015. pp. 1-5.

6. Odnokopylov G.I., Rozaev I.A. Fault-tolerant control of switched-reluctance drive in emergency modes. 2015 International Siberian Conference on Control and Communications (SIBCON): Proc. Omsk, May 21-23, 2015. pp. 1-6.

7. Odnokopylov G.I., Rozaev I.A. Fault-tolerant control algorithms of switched-reluctance motor drive in open-phase modes. 2016 The $11^{\text {th }}$ International Forum on Strategic Technology (IFOST): Proc. Novosibirsk, Jun 1-3, 2016. pp. 140-144.

8. Tkachuk R.Y., Glazyrin A.S. Principle of building a fault-tolerant control system for asynchronous electric drive. Bulletin of the Tomsk Polytechnic University, 2012, vol. 321, no. 5, pp. $105-109$.
9. Almashakbeh A.S., Prus V., Zagirnyak M. Models for electric machine reliability prediction at variation of the condition of basic structural units. Przeglad elektrotechniczny, 2017, vol. 1, no. 93 , pp. 117-120.

10. Muravleva 0.0., Tyuteva P.V. Operation features of the improved induction motors in the variable speed drive of pump units. EUROCON 2009. St. Petersburg, May 18-23, 2009. pp. 703-708.

11. Muravleva 0.0. Resource saving prospects based on accuracy theory. EUROCON 2007 - The International Conference on Computer as a Tool. Warsaw, Poland, September 9-12, 2007. pp. $1845-1850$.

12. Glowacz A., Glowacz Z. Diagnostics of stator faults of the singlephase induction motor using thermal images, MoASoS and selected classifiers. Measurement, 2016. vol. 93, pp. 86-93.

13. Zappalá D., Sarma N., Djurović S., Crabtree C.J., Mohammad A., Tavner P.J. Electrical \& mechanical diagnostic indicators of wind turbine induction generator rotor faults. Renewable energy, 2019, vol. 131, pp. 14-24.

14. Sakhara S., Saad S. Diagnosis and detection of short circuit in asynchronous motor using three-phase model. International journal of system assurance engineering and management, 2017, vol. 8, pp. 308-317.

15. Ma M., Wang R., Li F., Wang J., Yang S. A fault-tolerant control strategy for switched reluctance motor drive for electric vehicles under short-fault condition. Microelectronics reliability, 2018, vol. 88-90, pp. 1221-1225.

16. Tousizadeh M., Che H. S., Selvaraj J., Rahim N. A., Ooi B.-T. Performance comparison of fault-tolerant three-phase induction motor drives considering current and voltage limits. IEEE Tran- 
sactions on Industrial Electronics, 2019, vol. 66, no. 4 pp. $2639-2648$.

17. Shevchuk V.A., Muravlev 0.P. Analysis of failure-free operation probability for electrical machines in the diamond industry. $M i$ ning equipment and electrical engineering, 2018, vol. 4, no. 138, pp. 39-46. In Rus.

18. Zhiyong Z., Fei Z., Zhiqiang L., Xin Z. Brushless DC motor torque estimation based on nonlinear partial least square method. Tran sactions of China electrotechnical society, 2017, vol. 32, pp. $62-69$.

19. Zeng G., Yang X., Zhao S., Yin H., Pei Y., Cao J. Analysis, design and dynamic simulation of novel limited swing angle torque permanent magnet motor for high voltage circuit breaker application. Energies, 2018, vol. 11, no. 10, pp. 1-18.

20. Meng L., Liu Z. Fault simulation and diagnosis for vector control of AC motor drive. IEEJ Transactions on Electrical and Electronic Engineering, 2016, vol. 11, no. 5, pp. 618-623.

21. Zyuzev A.M., Bubnov M.V. Model for Sucker-Rod Pumping Unit Operating Modes Analysis Based on SimMechanics Library. Journal of Physics: Conference Series, 2018, vol. 944, pp. 1-7.

22. Zyuzev A.M., Metelkov V.P. Problem of induction motors rotor winding overheating in starting modes of high inertia electric drives of oil and mining industry. Bulletin of the Tomsk Polytechnic University. Geo Assets Engineering, 2018, vol. 329, no. 7, pp. 96-103. In Rus.

23. Zyuzev A.M., Metelkov V.P. Accounting temperature fluctuations in stator winding of induction motor when checking for heating in intermittent mode. Bulletin of the Tomsk Polytechnic University. Geo Assets Engineering, 2015, vol. 326, no. 4, pp. $146-153$.

24. Zyuzev A.M., Kostylev A.V., Stepanyuk D.P. A soft-startup device for an induction motor with supply-network voltage control. Russian Electrical Engineering, 2014, vol. 85 (9), pp. 586-590.

25. Ruan L., Chen J., Gu G. Different Influence of Cooling Method to Stator Bar Insulation Characteristics in Pumped Storage Units. Diangong Jishu Xuebao/Transactions of China Electrotechnical Society, 2017, vol. 32, no. 14, pp. 246-251.

26. Shevchuk V.A., Muravlev 0.P., Stolyarova 0.0. Survival analysis of a.c. machines in the diamond industry using the KaplanMeier estimator. MATEC Web of Conferences, 2017, vol. 141, pp. 1-4.

27. Shevchuk V.A., Muravlev 0.P., Stolyarova 0.0., Shevchuk V.P. The use of a special software for induction motor diagnostics in the diamond industry. MATEC Web of Conferences, 2017, vol. 91, pp. 1-4.

28. Saaty T. The Analytic Hierarchy Process: Planning, Priority Setting, Resource Allocation. New York, McGraw-Hill Publ., 1993. $320 \mathrm{p}$.

29. Saaty T.L., Vargas L.G. Models, Methods, Concepts \& Applications of the Analytic Hierarchy Process. Boston, Springer US, 2001. $333 \mathrm{p}$.

30. Andreychikov A.V., Andreychikova 0.N. Analiz, sintez, planirovanie resheniy $v$ ekonomike [Analysis, synthesis, planning decisions in the economics]. Moscow, Finansy i statistika Publ., 2000. $368 \mathrm{p}$.
31. Peregudov F.I., Tarasenko F.P. Osnovy sistemnogo analiza [Basics of system analysis]. Toms, NTL Publ., 2001. 396 p.

32. Brunelli M. Introduction to the Analytic Hierarchy Process. New York, Springer, 2015. 83 p.

33. Antonov A.V. Sistemny analiz [System analysis]. Moscow, Vysshaya shkola Publ., 2006. 454 p.

34. Tutygin A.G., Korobov V.B. Preimushchestva i nedostatki metoda analiza ierarkhiy [Advantages and disadvantages of the hierarchy analysis method]. Izvestia: Herzen University Journal of Humanities \& Sciences, 2010, no. 122, pp. 108-115.

35. Deorditsa Yu.S. Intellektualnye sistemy podderzhki prinyatiya resheniy [Intelligent Decision Support Systems]. Lugansk, VNU Publ., 2005, $64 \mathrm{p}$.

36. Chernysheva T.Y., Korchuganova M.A., Borisov V.V., Minkov S.L. Hierarchical model of assessing and selecting experts. IOP Conference Series: Materials Science and Engineering, 2016, vol. 127, pp. 1-6.

37. He M.X., An X. Information security risk assessment based on analytic hierarchy process. Indonesian Journal of Electrical Engineering and Computer Science, 2016, vol. 1, no. 3, pp. 656-664.

38. Gusev V.V., Muravlev 0.P., Shevchuk V.P. System analysis of the performance of electric machines in the mining complex. Bulletin of the Tomsk Polytechnic University, 2009, vol. 314, no. 4, pp. 74-78.

39. Fei S., Xiangdong L., Yali F. Safety evaluation for bridge crane based on FTA and AHP. MATEC Web of Conferences, 2018, vol. 207.

40. Makarova I.L. Analiz metodov opredeleniya vesovykh koeffitsientov $\mathrm{v}$ integralnom pokazatele obshchestvennogo zdorovya [Analysis of methods for determining weights in the integral index of public health]. Simvol nauki, 2015, no. 7, pp. 87-95.

41. Emrouznejad A., Ho W. Fuzzy Analytic Hierarchy Process. Boca Raton, CRC Press Publ., 2018. 407 p.

42. Greco S., Ehrgott M., Figueira J.R. Multiple Criteria Decision Analysis: State of the Art Surveys. New York, Springer Publ., 2016. $1346 \mathrm{p}$.

43. Kou G., Chao X., Peng Y., Xu L., Chen Y. Intelligent collaborative support system for AHP-group decision making. Studies in Informatics and Control, 2017, vol. 26, no. 2, pp. 131-142.

44. Ma X.D., Sun Q., Zhang J.M., He P. Research on comprehensive evaluation system of urban energy data based on AHP method. IOP Conference Series: Materials Science and Engineering, 2018, vol. 382 , no. 5, pp.1-7.

45. Aşchilean I., Badea G., Giurca I., Naghiu G.S., Iloaie F.G. Choosing the Optimal Technology to Rehabilitate the Pipes in Water Distribution Systems Using the AHP Method. Energy Procedia, 2017, vol. 112, pp. 19-26.

46. Evans G.W. Multiple Criteria Decision Analysis for Industrial Engineering: Methodology and Applications. Boca Raton, CRC Press Publ., 2018. 467 p.

47. Khazaii J. Advanced Decision Making for HVAC Engineers: Creating Energy Efficient Smart Buildings. Switzerland, Springer International Publ., 2016. 191 p.

Received: 30 January 2019.

\section{Information about the authors}

Georgy I. Odnokopylov, Dr. Sc., associate professor, National Research Tomsk Polytechnic University.

Yury N. Dementyev, Cand. Sc., professor, National Research Tomsk Polytechnic University.

Vladislav A. Shevchuk, postgraduate student, National Research Tomsk Polytechnic University. 